\title{
FACTORES QUE MODIFICAN LOS NIVELES DE HORMONAS TIROIDEAS EN AVES DOMÉSTICAS
}

\author{
JOSÉ HENRY OSORIO ${ }^{1}$ \\ LINA MARÍA URQUIJO ${ }^{2}$ \\ DIANA MARCELA SALAMANCA ${ }^{2}$ \\ jose.osorio_o@ucaldas.edu.co
}

Manizales, 2011-06-07 (Rev. 2011-08-23)

\section{RESUMEN}

Objetivo: analizar los factores que modifican los niveles de hormonas tiroideas en aves domésticas. Materiales y métodos: se analizó la literatura disponible de los últimos 50 años en las bases de datos BBCSLILACS, Fuente Académica, IB-PsycINFO, IB-SSCI, IB-SciELO, Scopus y Scirus, al igual que artículos históricos, textos y referencias citadas en trabajos públicos. Resultados: se obtuvo información pertinente relacionada con los objetivos propuestos en la presente revisión, por lo cual puede clasificarse en 2 secciones a saber: síntesis, liberación y metabolismo de hormonas tiroideas; factores que modifican los niveles de hormonas tiroideas. Conclusión: la liberación de hormonas tiroideas puede ser afectada por factores tales como temperatura, incubación, dieta, ayuno, producción, fotoperiodos y estrés.

\section{PALABRAS CLAVE:}

Aves, hormona tiroidea, metabolismo.

\section{FACTORS WHICH MODIFY THYROID HORMONES LEVELS IN DOMESTIC BIRDS}

\section{ABSTRACT}

Objective: to analyze the factors which modify the thyroid hormone levels in domestic birds. Materials and methods: information from the last 50 years included in the BBCS-LILACS, Fuente Académica, IB-PsycINFO, IBSSCI, IB-SciELO, Scopus and Scirus, data bases as well as historical articles, texts and references cited in work published to date were analyzed. Results: important information related to the objectives proposed in the present review was found and analyzed. The information was later divided into two sections as follows: synthesis, liberation and metabolism of thyroid hormones and factors which modify the thyroid hormones levels. Conclusion: thyroid hormones production can be influenced by factors such as temperature, incubation, diet, fasting, photoperiods and stress.

\section{KEY WORDS:}

Poultry, thyroid hormone, metabolism.

Abreviaturas: $\mathrm{T}_{3}$, triyodotironina; $\mathrm{T}_{4}$, tetrayodotironina; $\mathrm{TSH}$ (hormona estimulante de tiróides); TGB (tiroglobulina). 


\section{INTRODUCCIÓN}

En las aves comerciales, la glándula tiroidea inicia su desarrollo al segundo día de la etapa embrionaria que tiene un promedio de duración de 21 días. Se origina de la pared ventral de la faringe, asumiendo su posición definitiva aproximadamente al quinto día de la incubación. En las aves es un órgano par, de forma ovalada (Anne McNabb, 1999) que se ubica a cada lado de la tráquea en la entrada al tórax sin poseer istmo de conexión, se encuentra irrigada por la arteria tiroidea craneal y la caudal, que son ramas de la arteria carótida común. El retorno venoso se lleva a cabo por las venas tiroideas, que convergen en la vena yugular (Lumeij, 1994). Esta glándula comprende el $0,01-0,05 \%$ del peso corporal, la cual se relaciona con el crecimiento durante y después de la etapa embrionaria (Anne McNabb, 1999).

El epitelio de la glándula tiroidea posee dos tipos de células: las de revestimiento folicular $(90 \%)$ y las para-foliculares $(10 \%)$ (Banks, 1986) las cuales secretan calcitonina (Dickson et al., 2003). El epitelio glandular dispone sus células en círculos a los que se les conoce como folículos, estos contienen un material proteico homogéneo denominado coloide, que es la forma de almacenamiento de las hormonas tiroideas (Lumeij, 1994). Por exocitosis desde las células apicales se libera en el coloide la tiroglobulina, molécula producida en los ribosomas del retículo endoplásmico de las células foliculares (Anne McNabb, 1999). Para que desde la tiroides se dé la liberación hormonal se requiere que las células foliculares realicen endocitosis de las gotas de coloide, fusión con los lisosomas, digestión de tiroglobulinas por enzimas lisosomales y liberación de $T_{4}$ y $T_{3}$ a los capilares en la superficie externa de las células del folículo (Anne McNabb, 1999).

En la síntesis de las hormonas tiroideas participan dos tipos de moléculas: la tirosina y el yodo. La tirosina forma parte de una molécula de gran tamaño denominada tiroglobulina (TGB), que es formada en la célula folicular y posteriormente secretada a la luz del folículo (Dickson et al., 2003). La tiroglobulina contiene en su estructura carbohidratos (galactosa, manosa, $\mathrm{N}$-acetil-glucosamina, ácido siálico) y yodo-aminoácidos entre los que se incluyen a la 3-monoyodotirosina (MIT), a la 3-5-diyodotirosina (DIT), 3,5,3'-triyodotirosina ( $\left.T_{3}\right)$ y a la 3,5,3',5'-tetrayodotirosina ( $T_{4}$ ) (Banks, 1986). La TSH es una glicoproteína producida por tirotropos (células de la adenohipofisis) (Anne McNabb, 1999), actúa mediante el inicio de la formación de adenosína 3',5'-monofosfato cíclico (AMPc) y la fosforilación de las proteínas cinasas. La secreción de TSH está regulada por la retroalimentación negativa de la $\mathrm{T}_{3}$ y $\mathrm{T}_{4}$ que inhiben la síntesis de la hormona liberadora de tirotropina en el hipotálamo, además de inhibir la actividad de la TSH en la hipófisis (Dickson et al., 2003). Actúa directamente sobre las células glandulares de la tiroides desencadenando la secreción de tiroxina (T4) y triyodotironina ( $T_{3}$ ) (Lumeij, 1994).

\section{SÍNTESIS, LIBERACIÓN Y METABOLISMO DE HORMONAS TIROIDEAS}

La glándula tiroidea aviar está bajo el control del eje hipotálamo-pituitariotiroideo (HPT) el cual ejerce su control entre los días 10,5 y 11,5 de la etapa embrionaria. El hipotálamo produce dos hormonas: hormona liberadora de tirotropina y somatostatina que tienen efectos inhibitorios y estimulatorios, respectivamente sobre la pituitaria. Para que la síntesis de las hormonas se lleve a cabo, la glándula debe disponer de yodo (elemento halógeno) el cual se obtiene de la dieta, esta ingesta de yodo es proporcional al contenido de yoduro en la glándula tiroidea, pero los 
niveles de hormonas tiroideas circulantes y las que se encuentran en la glándula, no se ven alteradas por la misma (Anne McNabb, 1999). El yodo se convierte en yoduro en el intestino, desde el cual se transporta vía sanguínea a la glándula tiroidea, donde las células foliculares mediante un proceso de transporte activo lo atrapan (Dickson et al., 2003). La captación de yoduro por la tiroides aviar sucede en horas y su retención es prolongada, lo cual se encuentra influenciado por la cantidad de yoduro presente en la dieta (Anne McNabb, 1999). El yoduro atraviesa la pared apical de la célula donde se une a las estructuras anulares de la molécula de tirosina, estas estructuras pueden incorporar hasta dos moléculas de yoduro.

Se denomina monoyodotirosina cuando se une una molécula de yoduro, y diyodotirosina si se unen dos moléculas. La unión de dos moléculas de tirosina yodada forma las hormonas tiroideas de la siguiente manera: dos moléculas de diyodotirosina (DIT) forman tetrayodotironina $\left(T_{4}\right)$, y una molécula de monoyodotirosina (MIT) y una de diyodotirosina (DIT) forman triyodotironina $\left(T_{3}\right)$; en esta síntesis participa la enzima tiroperoxidasa en conjunto con el oxidante peróxido de hidrógeno, encargado de catalizar la yodación de los residuos de la globulina fijadora de tiroxina o tiroglobulina (TBG) y la formación de $\mathrm{T}_{3}$ y $\mathrm{T}_{4}$ (Dickson et al., 2003). El acoplamiento de estas moléculas se lleva a cabo bajo la influencia de la TSH o tirotropina (Banks, 1986). La acción de las hormonas tiroideas, tanto en las aves como en los mamíferos, requiere la presencia de receptores nucleares. Se les conoce como receptores $\mathrm{T}_{3}$ por su mayor afinidad con esta hormona (Anne McNabb, 1999). La deyodación y la conjugación son las principales vías metabólicas de las hormonas tiroideas (Reyns et al., 2002). La tiroxina secretada por la glándula tiroides es convertida a triyodotironina para que pueda ejercer su actividad biológica, esta activación se lleva a cabo en los tejidos periféricos por la enzima yodotironina deyodasa (D) que permite la deyodación del anillo externo (ORD) de la molécula.

Hasta el momento se conocen tres tipos de deyodasas (D1, D2, D3), estas enzimas tienen como función convertir tiroxina en 3,3'-diyodotironina (3,3'$\mathrm{T}_{2}$ ) y triyodotironina en 3,3',5'-triyodotironina $\left(\mathrm{rT}_{3}\right)$ al deyodar el anillo interno (IRD). La D1 está involucrada en la deyodación del anillo interno y el externo, la D2 por medio de la deyodación del anillo externo convierte $\mathrm{T}_{4}$ y $\mathrm{rT}_{3}$ y la $\mathrm{D} 3$ es capaz de inactivar la $\mathrm{T}_{4}$ y $\mathrm{T}_{3}$ por la deyodación del anillo interno (Reyns et al., 2002; Decuypere, 2005). La conjugación de las hormonas tiroideas comprende la sulfatación o glucuronidación del grupo hidroxifenólico. Este proceso permite la desintoxicación e incrementa la solubilidad de las hormonas tiroideas en el agua para facilitar su excreción urinaria y biliar. La sulfatación bloquea la función de la D2 y D3 mientras incrementa la inactivación por parte de D1; cuando esta última se encuentra disminuida los sulfatos conjugados no se degradan, lo cual protege a los tejidos del exceso de las hormonas tiroideas activas (Reyns et al., 2002; Decuypere, 2005).

\section{FACTORES QUE MODIFICAN LOS NIVELES DE HORMONAS TIROIDEAS}

\section{Temperatura}

En las aves comerciales se ve afectado el metabolismo por las bajas temperaturas, siendo la temperatura letal para un pollito de un día de $15,5^{\circ} \mathrm{C}$ y para las aves adultas de $22,2^{\circ} \mathrm{C}$ (Julian, 2005). Durante los primeros días de vida, el metabolismo se enlentece debido a que la aves no son capaces de mantener la temperatura corporal; en las aves adultas dichas temperaturas ocasionan aumento en el metabolismo en un intento 
por conservar la temperatura normal, ocasionando hipertensión pulmonar y en ambos casos la muerte (Julian et al., 1989). Las aves que son sometidas a bajas temperaturas durante cortos periodos de tiempo presentan un aumento en la $T_{3}$ plasmática, mientras que los periodos largos no ejercen ningún efecto sobre esta, lo que se debe a una mejora en la conversión de $T_{4}$ a $T_{3}$, proceso que es sensible a la temperatura ambiental. La $\mathrm{T}_{4}$ plasmática no se ve alterada por dichas temperaturas y periodos (Hangalapura et al., 2004). Por otro lado, las altas temperaturas afectan al embrión durante su desarrollo, causando anormalidades o la muerte; esto puede suceder en cualquier etapa del ciclo productivo. La producción de calor corporal está directamente afectada por la masa corporal y la ingesta de alimento, pero puede variar con la temperatura ambiental, siendo letal la temperatura corporal de $46,6^{\circ} \mathrm{C}$ para pollitos y de $47,2^{\circ} \mathrm{C}$ para aves adultas. En altas temperaturas la tasa metabólica de los pollos de engorde aumenta, debido a que el animal gasta energía en un intento por regular su temperatura corporal, además aumenta la pérdida de agua llevando a deshidratación y/o muerte (Julian, 2005).

Las aves regulan su secreción de hormonas tiroideas según la temperatura ambiental a la que se encuentran sometidas, de esta manera, si la temperatura aumenta la secreción disminuye y viceversa (Silva, 2003). Así por ejemplo, en pollos de engorde sometidos a estrés calórico el primer día el nivel de $\mathrm{T}_{3}$ disminuye y al siguiente día hay una disminución de $\mathrm{T}_{4}$ (Tao et al., 2006). Otros estudios han mostrado que los pollos de engorde, codornices y pavos que son sometidos a estrés calórico aumentan sus niveles de $\mathrm{T}_{3}$ y $\mathrm{T}_{4}$, demostrando así que las líneas de engorde responden de manera diferente a las condiciones ambientales; lo que parece explicar este hecho, es que las aves comerciales al tener un mayor tamaño corporal se les dificulta la adecuada disipación del calor comparado con aves sin selección genética. Una respuesta de las hormonas tiroideas constante a través del tiempo en aves sometidas a cambios en la temperatura ambiental, permite la obtención de una mejor calidad de carne, ya que estas están involucradas en la regulación del calcio en el musculo esquelético (Chiang et al., 2008).

\section{Incubación}

La manipulación térmica durante la embriogénesis ocasiona una disminución de la concentración plasmática de $T_{3}$, por una reducida deyodación periférica de $\mathrm{T}_{4}$, principalmente en el hígado, y el aumento en el catabolismo de $\mathrm{T}_{3}$, ocasionando un bajo rendimiento del pollo, aunque al ser expuestos al calor mejora su capacidad termorreguladora (Piestun et al, 2008). En el proceso de incubación del pollo de engorde, se demostró que entre los días 17 al 20 luego de ser sometidos a temperaturas entre 36 y $39^{\circ} \mathrm{C}$ se presenta una disminución en las concentraciones de las hormonas tiroideas, mientras que al ser sometidos a diferentes concentraciones de oxígeno (17, 19, 21 y 23\%) dichas hormonas aumentan (Wineland et al., 2006). Ha sido demostrado que los huevos incubados a altas temperaturas presentan disminución en los niveles plasmáticos de $T_{3}$ post-eclosión; también se observa que los pollos cuyos huevos son sometidos a altas temperaturas durante la incubación, tienen mayor tolerancia a altas temperaturas ambientales, lo que genera cambios en las hormonas tiroideas ( $\left.T_{3}\right)$ (Yalçın et al., 2009). Los huevos que son incubados a grandes altitudes $(1800 \mathrm{msnm})$ durante los primeros 10 días, presentan un aumento en los niveles séricos de $T_{3}$ y $T_{4}$ en los días 10 y 19 de incubación. Se conoce que durante la incubación estas hormonas participan en el cambio de la respiración alantoidea a pulmonar y, además, en la duración de la incubación (Bahadoran et al., 2010).

Los niveles plasmáticos de $\mathrm{T}_{4}$ se elevan hasta por 300 minutos postinyección, cuando se inyectan $5 \mu \mathrm{g}$ de TRH en huevos de pavo con 24 días de incubación, además se ha demostrado que la TRH mejora la 
incubabilidad (Christensen \& Phelps, 2001). Las bajas temperaturas durante la incubación, hacen que se alargue este proceso, mientras que las altas temperaturas lo aceleran. El día de la eclosión los huevos que presentan una perforación en la cámara de aire tienen una elevación sérica de $T_{3}$ mas no de $T_{4}$ comparados con los huevos que no poseen dicha perforación, esto evidencia el importante papel de la $T_{3}$ en la ruptura y eclosión (Decuypere et al., 1979). Los huevos que son almacenados durante tres días tienen valores plasmáticos embrionarios de $T_{3}$ más altos que los almacenados por 18 días, y cuando nacen son los niveles de $\mathrm{rT}_{3}$ los que se encuentran aumentados (Tona et al., 2003).

\section{Dieta}

El yodo es esencial para la formación de hormonas tiroideas, su deficiencia y/o exceso conllevan a la presentación de bocio (aumento de la glándula tiroides) tanto en aves como en mamíferos. Las manifestaciones clínicas de esta afección están directamente relacionadas con problemas respiratorios, como resultado de la presión ejercida por la glándula sobre la tráquea (Macwhirter, 1994). Dietas ricas en yodo suministradas a hembras de pavos, generan una disminución en el $\mathrm{T}_{4}$ plasmático del embrión, acompañada de una reducida tasa de supervivencia (Christensen et al., 2002). Se ha observado que las hembras suplementadas con 4 ppm de yodo, muestran una disminución en las concentraciones sanguíneas de $\mathrm{T}_{4}$, y una mejora en la tasa de supervivencia de los embriones. También se ha evidenciado un incremento en las concentraciones sanguíneas de $\mathrm{T}_{4}$ entre el día 25 al 28 de incubación, mientras que las concentraciones sanguíneas de $T_{3}$ disminuyen en el día 27 de incubación. Lo anterior, indica que la función tiroidea del embrión durante la eclosión se ve influenciada por el suministro de yodo en la dieta materna (Christensen \& Davis, 2004).

Las dietas deficientes en selenio producen alteraciones en el metabolismo de las hormonas tiroideas, debido a que las deyodasas tipo I, II y III son enzimas dependientes de selenio. Al existir una deficiencia de este elemento, se produce una disminución plasmática en los niveles de $T_{3}$, mientras que los niveles de $\mathrm{T}_{4}$ se incrementan. Los niveles de estas hormonas no se ven afectados en dietas deficientes en vitamina $E$ (Chang et al., 2005). Por otro lado, una dieta deficiente en fosforo $(0,05$ y $0,10 \%)$ ocasiona una disminución en los niveles séricos de $T_{3}$ y $T_{4}$, aunque en este último no son constantes. Esta deficiencia conlleva a un crecimiento más lento y una menor ingesta de alimento (Parmer et al., 1987). Con una deficiencia de $0,14 \%$ en machos de la línea Leghorn las concentraciones plasmáticas de $T_{3}$ y $T_{4}$ no tuvieron un cambio significativo, pero la $T_{4}$ tendió a estar baja (Carew et al., 1985).

Con relación a los niveles de proteína en la dieta, se ha encontrado que las gallinas ponedoras alimentadas con una dieta a base de maíz y soya poseen una concentración plasmática de $T_{4}$ y $T_{3}$ más elevada que las aves que son alimentadas con harina de pescado (Akiba et al., 1982). Dietas con niveles de proteína menor al $24 \%$ generan un aumento de los niveles plasmáticos de $\mathrm{T}_{3}$ y una diminución en los de $\mathrm{T}_{4}$ (Keagy et al., 1987; Carew \& Alster, 1997). El uso de una dieta baja en proteína y energía produce valores de $\mathrm{T}_{3}$ y $\mathrm{GH}$ aumentados y los de $\mathrm{T}_{4}$ disminuidos, cuando se comparan con dietas formuladas con alta proteína y energía (Moravej et al., 2006). Normalmente la $T_{3}$ plasmática sufre un aclaramiento más rápido que la $T_{4}$ debido a que no requiere unirse a proteínas plasmáticas; por tal motivo, al haber deficiente proteína en la dieta la $\mathrm{T}_{4}$ no puede unirse a estas, con lo cual se altera el proceso disminuyéndose así sus niveles plasmáticos (Hutchins \& Newcomer, 1966). Otra explicación probable de este hecho, es que la $T_{4}$ al no convertirse en $\mathrm{T}_{3}$ genera un aumento de la $\mathrm{rT}_{3}$ (metabolito inactivo de $\mathrm{T}_{4}$ ), ya que el efecto fundamentalmente es observado durante una restricción 
de energía (Keagy et al., 1987). Por otro lado, los altos niveles de proteína cruda en la dieta ocasionan un aumento en la $\mathrm{T}_{4}$ plasmática y una disminución en la $\mathrm{T}_{3}$ (Rosebrough, 2000).

Una dieta deficiente en triptófano disminuye los niveles plasmáticos de $T_{4}$ y $\mathrm{rT}_{3}$ a las dos semanas, por el contrario los niveles de $\mathrm{T}_{3}$ aumentan; esto último probablemente se debe a la deficiencia del aminoácido en sí, mas no a una deficiencia proteica. También puede ser causado por una cantidad reducida de 5-hidroxitriptamina o 5-HT en los tejidos, pues se sabe que inhibe la síntesis de la hormona tiroidea (Carew et al., 1983). La deficiencia de lisina en la dieta no reduce los niveles séricos de $\mathrm{T}_{3}$ pero sí afecta los niveles de $T_{4}$, disminuyéndolos. Una dieta deficiente en fenilalanina y tirosina ocasionan una disminución significativa en los niveles séricos de $T_{3}$, comparado con dietas ricas en todos los aminoácidos y con deficiencias en lisina (Elkin et al., 1980; Carew et al., 1997). Por otro lado dietas con 0,88 y $0,66 \%$ de lisina, elevan la concentración plasmática de $\mathrm{T}_{3}$, mientras que en dietas con una mayor deficiencia $(0,53 \%)$ los valores permanecen estables, la $\mathrm{T}_{4}$ no presenta cambios (Carew et al., 2005). Al existir una deficiencia de cualquier aminoácido esencial, se limita la síntesis de tiroglobulina (glicoproteína) la cual es esencial para la síntesis de las hormonas tiroideas, lo que explicaría la disminución de la $T_{3}$ y $T_{4}$ en la circulación (Elkin et al., 1980). Una deficiencia treonina no afecta los niveles circulantes de hormonas tiroideas (Carew et al., 1997). La deficiencia de metionina $(0,25 \%)$, sin embargo, causa una elevación plasmática de los niveles de $\mathrm{T}_{3}$ (Carew et al., 1997). Esto ha sido comprobado usando diferentes niveles de este aminoácido $(0,4,0,3$ y 0,2\%) (Carew et al., 2003). Suministrados en exceso al pollo de engorde, 10 aminoácidos esenciales (histidina, isoleucina, fenilalanina, triptófano, valina, arginina, leucina, lisina, metionina, treonina), se obtuvo como resultado que dos de ellos (isoleucina y valina) generan un aumento en la $T_{3}$ plasmática, mientras los niveles de $T_{4}$ no se ven alterados (Carew et al., 1998).

\section{Ayuno}

Tanto en aves como en mamíferos el eje tiroideo sufre cambios si es sometido a restricción alimenticia o ayuno, provocando que las concentraciones plasmáticas de $T_{3}$ disminuyan y las de $T_{4}$ aumenten (Darras et al., 1995; Geris et al., 1999; Buyse et al., 2002; Reyns et al., 2002). En hembras de la línea Hybro se ha comprobado que los niveles $T_{3}$ disminuyen con la edad, mientras que los de $\mathrm{T}_{4}$ aumentan en el mismo periodo (Bruggeman et al., 1997). Los cambios en la $T_{3}$ plasmática son causados por la deyodación hepática del anillo interno, mientras que las fluctuaciones de $\mathrm{T}_{4}$ plasmática no se pueden correlacionar con las periféricas (Darras et al., 1995; Geris et al., 1999). Estos cambios son debidos a que durante el ayuno la actividad de las deyodasas varía; la D1 no presenta una fluctuación significativa en condiciones de restricción alimenticia, en contraste la actividad de la D2 disminuye y la D3 se incrementa significativamente (Györffy et al., 2009). La concentración hepática de $T_{3}$ disminuye en animales sometidos a ayuno y se regulariza entre 120 y 200 minutos después de suministrarles el alimento nuevamente. En contraste, las concentraciones hepáticas de $T_{4}$ aumentan durante el ayuno y se mantienen elevadas después de la realimentación (Reyns et al., 2002). En aves sometidas a ayuno, los niveles de $\mathrm{TRH}_{\mathrm{y}} \mathrm{T}_{4}$ tienden a aumentar, mientras que las concentraciones de $\mathrm{T}_{3}$ y $\mathrm{TSH}$ disminuyen (Geris et al., 1999).

\section{Producción}

Durante el pico de producción a la semana 26 y la muda a la semana 64 , los niveles plasmáticos de $T_{3}$ disminuyen; mientras que los niveles de $T_{4}$ en la semana 51 (pico de producción) disminuyen y en la 64 (muda) 
aumentan. Al aumentar la densidad de población los valores de las hormonas tiroideas no se ven afectados (Davis et al., 2000). Durante el proceso de muda a hembras de pavos se les suministró una dieta con hormonas tiroideas o tiuracilo (bloqueante tiroideo), las que fueron suplementadas con $\mathrm{T}_{4}$ redujeron el tiempo para retornar a la producción de huevos, mientras que aquellas suplementadas con $T_{3}$ o tiuracilo presentaron un aumento en dicho tiempo. Los bajos niveles de $T_{4}$ mas no los de $T_{3}$ pueden alargar el periodo de muda (Queen et al., 1997).

Al llevarse a cabo el periodo de muda, las concentraciones plasmáticas de $\mathrm{T}_{3}$ y $\mathrm{T}_{4}$ por lo general son bajas, esto muestra que las hormonas tiroideas están involucradas en los procesos fisiológicos de la finalización de la postura y la muda en hembras de pavo (Lien \& Siopes, 1993). En hembras de pavo de un año, sometidas a fotoperiodos cortos (8 horas de luz), no se observan cambios significativos en las concentraciones plasmáticas de $T_{3}$, mientras que al exponerlas a fotoperiodos largos (16 horas de luz) la $T_{3}$ aumenta, mientras que no se evidencian cambios en la $\mathrm{T}_{4}$ plasmática en ninguno de los fotoperiodos. Por otro lado, durante el replume las concentraciones plasmáticas de $\mathrm{T}_{3}$ aumentan, mas no cambian las de $\mathrm{T}_{4}$; esto demuestra la participación de la $\mathrm{T}_{3}$ al promover la fotosensibilización y disminuir la foto-refractariedad en la muda inducida por fotoperiodos cortos en pavos (Siopes, 2002). Se ha evidenciado que no es necesario un cambio en las hormonas tiroideas para que ocurra la fotosensibilización, debido a que los niveles hormonales son similares tanto en hembras sometidas a fotoperiodos largos como las que no son expuestas a fotoperiodos (Proudman \& Siopes, 2006). Luego de la fotosensibilización los niveles de T3 disminuyen y los de $\mathrm{T}_{4}$ aumentan (Proudman \& Siopes, 2002, 2005) y permanecen estables, la $T_{3}$ a partir de la semana 12 de estimulación se incrementa lentamente en machos alimentados a voluntad. Las concentraciones plasmáticas de $T_{3}$ y $T_{4}$ en hembras y machos sometidos a fotosensibilización y con una restricción alimenticia se mantienen estables en hembras, mientras en machos varían semana a semana (Proudman \& Siopes, 2005).

\section{Otros factores}

Durante el periodo de oscuridad la liberación hormonal desde la glándula tiroidea es mucho mayor, aunque la conversión extratiroidea de $T_{4}$ a $T_{3}$ se eleva en el periodo de luz. El pico en la concentración plasmática de $T_{4}$ ocurre en el periodo de oscuridad, mientras que el de la $T_{3}$ se da en el de luz (Anne McNabb, 1999). Asimismo durante el estrés, el cual puede ser causado por múltiples factores como el aislamiento, cambios climáticos, miedo, hambre y manejo, se observa aumento en los niveles de corticosterona la cual afecta el eje tiroideo, induciendo una disminución en las concentraciones plasmáticas de TSH (Geris et al., 1999; Hudelson \& Hudelson, 2009).

\section{CONCLUSIÓN}

Existe una gran variedad de factores que pueden alterar la secreción normal de las hormonas tiroideas en las aves comerciales (Gutiérrez, 1999). Dichos factores, obran ocasionando disminuciones en los parámetros productivos o incluso pueden llevar a la muerte del animal. El conocimiento de cómo actúan dichos factores en el organismo animal, nos puede ayudar a manipular positivamente el ambiente en el que permanece el ave y, con ello, lograr una mejora en la producción (carne o huevos), en la supervivencia del animal y/o en su desarrollo como tal. 


\section{REFERENCIAS}

- Akiba, Y., Jensen, L. S., Barb, C. R., y Kraeling, R. R. (1982). Plasma estradiol, thyroid hormones, and liver lipid content in laying hens fed different isocaloric diets. J Nutr, 112, 299-9.

- Anne Mcnabb, F. M. (1999). Sturkie's avian physiology. 5 ed. USA: Academic Press. pp. 455-10.

- Bahadoran, S., Hassanzadeh, M., y Zamanimoghaddam, A. K. (2010). Effect of chronic hypoxia during the early stage of incubation on prenatal and postnatal parameters related to ascites syndrome in broiler chickens. Iranian J Vet Res, 11(1), 6471.

- Banks, W. J. (1986). Histología veterinaria aplicada. 1 ed. Ciudad de México: El Manual Moderno S.A. de C.V. pp. 583-8.

- Bruggeman, V., Vanmontfort, D., Renaville, R, Portetelle, D., y Decuypere, E. (1997). The effect of food intake from two weeks of age to sexual maturity on plasma growth hormone, insulin-like growth factor-I, insulin-like growth factor-binding proteins, and thyroid hormones in female broiler breeder chickens. Gen Comparat Endocrinol, 107, 212-8.

- Buyse, J., Janssens, K., Van der Geyten, S., Van As, P., Decuypere, E., y Darras, V. M. (2002). Pre- and postprandial changes in plasma hormone and metabolite levels and hepatic deiodinase activities in meal-fed broiler chickens. Brit $\mathrm{J}$ Nut, 88, 641-12.

- Carew, L. B., y Alster, F. A. (1997). Dietary carbohydrate and fat do not alter the thyroid response to protein deficiency in chicks (Abstract). Proc Soc Exp Biol Med, 215(1), 82-6.

- Carew, L. B. Jr., Alster, F. A., Foss, D. C., y Scanes, C. G. (1983). Effect of a tryptophan deficiency on thyroid gland, growth hormone and testicular functions in chickens. J Nutr, 113, 1756-9.

- Carew, L. B, Evarts, K. G, y Alster, F. A. (1997). Growth and plasma thyroid hormone concentrations of chicks fed diets deficient in essential amino acids. Poult Sci, 76, 1398-6.

- $\quad$ (1998). Growth, feed intake, and plasma thyroid hormone levels in chicks fed dietary excesses of essential amino acids. Poult Sci, 77, 295-3.

- Carew, L. B. Jr., Gestone, T. A., Alster, F. A. (1985). Effect of phosphorus deficiency on thyroid function and growth hormone in the white Leghorn male. Poult Sci, 64, 2010-2.

- Carew, L. B., McMurtry, J. P., y Alster, F. A. (2003). Effects of methionine deficiencies on plasma levels of thyroid hormones, insulin-like growth factors-I and -II, liver and body weights, and feed intake in growing chickens. Poult Sci, 82, 1932-6.

- $\quad$ Effects of lysine deficiencies on plasma levels of thyroid hormones, insulin-like growth factors I and II, liver and body weights, and feed intake in growing chickens. Poult Sci, 84, 10455 .

- Chang, W. P., Combs, G. F. Jr., Scanes, C. G., y Marsh, J. A. (2005). The effects of dietary vitamin $E$ and selenium deficiencies on plasma thyroid and thymic hormone concentrations in the chicken. Develop Comparat Immunol, 29, 265-8.

- Chiang, W., Booren, A., y Strasburg, G. (2008). The effect of heat stress on thyroid hormone response and meat quality in turkeys of two genetic lines. Meat Sci, 80, 615-7.

- Christensen, V. L., y Davis, G. S. (2004). Maternal dietary iodide influences turkey embryo thyroid function. Poult Sci, 3(9), 550-7.

- Christensen, V. L., Davis, G. S., y Nestor, K. E. (2002). Environmental incubation factors influence embryonic thyroid hormones. Poult Sci, 81, 442-8. 
- Christensen, V. L., y Phelps, P. (2001). Injection of thyrotrophinreleasing hormone in turkey embryos elevates plasma thyroxine concentrations. Poult Sci, 80, 643-3.

- Darras, V. M., Cokelaere, M., Dewil, E., Arnouts, S., Decuypere, E., y Kühn, E. R. (1995). Partial food restriction increases hepatic inner ring deiodinating activity in the chicken and the rat. Gen Comparat Endocrinol, 100, 334-4.

- Davis, G. S., Anderson, K. E., y Carroll, A. S. (2000). The effects of long-term caging and molt of single comb white leghorn hens on herterophil to lymphocyte ratios, corticosterone and thyroid hormones. Poult Sci, 79, 514-4.

- Decuypere, E., Nouwen, J., Kühn, E. R., Geers, R., y Michels, H. (1979). Lodohormones in the serum of chick embryos and posthatching chickens as influenced by incubation temperature. Relationship with the hatching process and thermogenesis. An Biol Anim Bioch Biophys, 19(6), 1713-10.

- Decuypere, E., Van Asa, P., Van der Geyten, S., y Darras, V. M. (2005). Thyroid hormone availability and activity in avian species: A review. Dom Anim Endocrinol, 29, 63-14.

- Dickson, W. M., Feldman, E. C., Hedge, G. A., Martin, R., y McDonald, L. E. (2003). Fisiología veterinaria cunningham. En Cunningham, J. G. (Ed,), Las glándulas endocrinas y su función (chapter 33, pp. 342-42). 3 ed. Madrid: Elsevier España S.A.

- Elkin R. G., Featherston, W. R., y Rogler, J. C. (1980). Effects of dietary phenylalanine and tyrosine on circulating thyroid hormone levels and growth in the chick. J Nutr, 110, 130-8.

- Geris, K. L., Berghman, L. R., Kühn, E. R., y Darras, V. M. (1999). The drop in plasma thyrotropin concentrations in fasted chickens is caused by an action at the level of the hypothalamus: role of corticosterone. Domestic Anim Endocrinol, 16(4), 231-6.

- Gutiérrez, G. (1999). Hormonas y reproducción en aves: la influencia de factores ambientales y sociales. Rev Lat Psicol, 31(1), 151-74.

- Győrffy, A., Sayed-Ahmed, A., Zsarnovszky, A., Frenyó, V. I., Decuypere, E, y Bartha, T. (2009). Effects of energy restriction on thyroid hormone metabolism in chickens. Acta Vet Hungarica, 57(2), 319-11.

- Hangalapura, B. N., Nieuwland, M. G. B., Buyse, J., Kemp, B., y Parmentier, H. K. (2004). Effect of duration of cold stress on plasma adrenal and thyroid hormone levels and immune responses in chicken lines divergently selected for antibody responses. Poult Sci, 83, 1644-5.

- Hudelson, K. S., y Hudelson, P. (2009). Endocrine considerations. En Harrison, G. J., y Lightfoot, T. L. (Eds.), Clinical avian medicine. Ithaca, NY: Internet Publisher, International Veterinary Information Service.

- Hutchins, M. O., y Newcomer, W. S. (1066). Metabolism and excretion of thyroxine and triiodothyronine in chickens (Abstract). Gen Compart Endocrinol, 6(2), 239-9.

- Keagy, E. M., Carew, L. B., Alster, F. A., y Tyzbir, R. S. (1987). Thyroid function, energy balance, body composition and organ growth in protein-deficient chicks. J Nutr, 117, 1532-8.

- Julian, R. J. (2005). Production and growth related disorders and other metabolic diseases of poultry: A review. Vet J, 169, 350-19.

- Julian, R. J., McMillan, I., y Quinton, M. (1989). The effect of cold and dietary energy on right ventricular hypertrophy, right ventricular failure and ascites in meat-type chickens. Avian Pathol, 18, 675-9.

- Lien, R. J., y Siopes, T. D. (1993). The relationship of plasma thyroid hormone and prolactin concentrations to egg laying, incubation behavior, and molting by female turkeys exposed to a 
one-year natural daylength cycle (Abstract). Gen Comparat Endocrinol, 90(2), 205-8.

- Lumeij, J. T. (1994). Endocrinology. En Ritchie, B. W., Harrison, G. J., y Harrison, L. R. (Eds.), Avian Medicine: Principles and Application (p. 582-606). Lake Worth, Florida: Wingers Publishing.

- Macwhirter, P. (1994). Malnutrition. En Ritchie, B. W., Harrison, G. J., y Harrison, L. R. (Eds.), Avian Medicine: Principles and Application (chapter 31, p. 859). Lake Worth, Florida. Wingers Publishing.

- Moravej, H., Khazali, H., Shivazad, M., y Mehrabani-Yeganeh, H. (2006). Plasma concentrations of thyroid hormone and growth hormone in lohmann male broilers fed on different dietary energy and protein levels. Int J Poult Sci, 5(5), 457-5.

- Parmer, T. G., Carew, L. B., y Alster, F. A. (1987). Thyroid function, growth hormone, and organ growth in broilers deficient in phosphorus. Poult Sci, 66, 1995-9.

- Piestun, Y., Shinder, D., Ruzal, M., Halevy, O., y Yahav, S. (2008). The effect of thermal manipulations during the development of the thyroid and adrenal axes on in-hatch and post-hatch thermoregulation. J Thermal Biol, 33, 413-5.

- Proudman, J. A., y Siopes, T. D. (2002). Relative and absolute photorefractoriness in turkey hens: profiles of prolactin, thyroxine, and triiodothyronine early in the reproductive cycle. Poult Sci, 81, 1218-5.

-

(2005). Thyroid hormone and prolactin profiles in male and female turkeys following photostimulation. Poult Sci, 84, 942 4.

- (2006). Potential role of thyroid hormones and prolactin in the programming of photorefractoriness in turkey hens. Poult Sci, 85, 1457-4.

- Queen, W. H., Christensen, V. L., y May, J. D. (1997). Supplemental thyroid hormones and molting in turkey breeder hens. Poult Sci, 76, 887-6.

- Reyns, G. E., Janssens, K. A., Buyseb, J., Kühn, E. R., y Darras, V. M. (2002). Changes in thyroid hormone levels in chicken liver during fasting and refeeding. Comparat Biochem Physiol, 132, 239-6.

- Rosebrough, R. W. (2000). Dietary protein levels and the responses of broilers to single or repeated cycles of fasting and refeeding. Nut Res, 20(69), 877-9.

- Silva, J. E. (2003). The thermogenic effect of thyroid hormone and its clinical implications. Ann Int Med, 139, 205-8.

- Siopes, T. D. (2002). Circulating thyroid hormone levels in recycled turkey breeder hens during a short day prelighting period and renewal of photosensitivity for egg production. Poult Sci, 81, 1342-4.

- Tao, X., Zhang, Z. Y., Dong, H., Zhang, H., y Xin, H. (2006). Responses of thyroid hormones of market-size broilers to thermoneutral constant and warm cyclic temperatures. Poult Sci, $85,1520-8$.

- Tona, K., Malheiros, R. D., Bamelis, F., Careghi, C., Moraes, V. M. B., Onagbesan, O., Decuypere, E., y Bruggeman, V. (2003). Effects of storage time on incubating egg gas pressure, thyroid hormones, and corticosterone levels in embryos and on their hatching parameters. Poult Sci, 82, 840-5.

- Wineland, M. J., Christensen, V. L., Yildrum, I., Fairchild, B. D., Ort, D. T., y Mann, K. M. (2006). Incubator environment interacts with genetic line of broiler at the plateau stage to affect embryo plasma thyroxine and triiodothyronine concentrations. Poult Sci, 5(8), 714-8. 
- Yalçın, S., Bruggeman, V., Buyse, J., Decuypere, E., Cabuk, M., y Siegel, P. B. (2009). Acclimation to heat during incubation: 4. blood hormones and metabolites in broilers exposed to daily high temperatures. Poult Sci, 88, 2006-7.

1. Laboratorio de Investigación en Bioquímica Clínica y Patología Molecular, Departamento de Ciencias Básicas de la Salud. Universidad de Caldas.

2. Departamento de Salud Animal, Universidad de Caldas. Correspondencia: jose.osorio_o @ucaldas.edu.co 